\title{
ORIGEN FLORAL DE LAS CARGAS DE POLEN RECOGIDAS POR APIS MELLIFERA L. EN ALORA (MALAGA, EsPAÑA)
}

\author{
Mª.Isabel HIDALGO, M.Lourdes BOOTELLO y Juan PACHECO
}

RESUMEN: El análisis microscópico de las cargas de polen recogidas por Apis mellifera en el período tradicional de cosecha de polen en la zona, nos revela 22 tipos polínicos, pertenecientes fundamentalmente a especies de las familias Cistaceae, Fabaceae y Rutaceae. Se relacionan los datos fenológicos, la frecuencia y el tipo de recurso ofrecido de las especies melitófilas, con los resultados obtenidos en los análisis polínicos.

Palabras clave: Melitopalinología, cargas de polen, fenología, Málaga, España.

ABSTRACT: The microscopical analysis of the pollen loads collected by Apis mellifera during the pollen harvesting has shown 22 pollen types The familys Cistaceae, Fabaceae and Rutaceae are the main pollen sources in the studied zone. Data on flowing fenology, frecuency and floral resources have been relationed with the pollen analysis.

Key words: Melissopalynology, pollen loads, flowering phenology, Málaga,Spain.

\section{N T R O D U C C I O N}

La abeja melífera vive en estrecha relación con la vegetación circundante, de la que obtiene para su alimentación el néctar y el polen, principalmente. Esta relación insecto-planta es interesante de conocer, por cuanto nos revela las preferencias alimenticias de la abeja. Así, conociendo al mismo tiempo la vegetación de una zona, podremos valorar el aprovechamiento apícola de un territorio, tanto para la obtención de determinados productos, como para la polinización de cultivos.

El polen, como fuente principal de proteínas y lípidos para las abejas, va a ser recogido con gran intensidad en el período primaveral, coincidiendo con el desarrollo de las larvas (Jean-Prost, 1989). En función de la floración disponible, las preferen- 
cias alimenticias de las abejas se manifiestan en la utilización de sólo una parte de la flora a su alcance, siendo ésta muy reducida en comparación con el número de especies que viven en la zona de pecoreo (Synge, 1947; Louveaux,1958b).

El análisis microscópico de las cargas de polen recolectadas durante un período determinado, nos puede orientar sobre qué plantas han sido utilizadas por las abejas como fuente de polen (Synge,1947; Percival, 1947, 1950, 1955; Louveaux, 1958c; Sharma, 1970). En España, se han iniciado recientemente trabajos de este tipo (González-Benavente, 1984; Serra \& col., 1986; Gómez-Ferreras 1986; Serra, 1988, etc).

La zona donde hemos realizado el presente trabajo (Alora, El Sabinal) se caracteriza por un matorral compuesto principalmente por Cistus albidus L., C. salviefolius L, Rosmarinus officinalis. L., Ulex baeticus Boiss., Adenocarpus telonensis (Loisel) DC., Lavandula stoechas L. Thymus mastichina L. y Thymbra capitata (L.) Cav. entre otras. Encontramos también parcelas dedicadas al cultivo de cereales, leguminosas, cítricos, almendros y olivos.

Siendo en dicha localidad la apicultura un tipo de explotación tradicional, se escogió el período primaveral para estudiar algunos aspectos de la recolección de polen por las abejas, con el fin de dislucidar qué fuentes poliníferas son las más importantes en la zona.

\section{A T E R I A L Y M E T O D O}

De un grupo de colmenas emplazadas en el área de estudio, se ha seleccionado una colmena (tipo Dadan't), en la que se ha instalado un cazapolen estándar. El período durante el cual se ha mantenido colocado ha sido desde el 25 de Marzo de 1989 hasta el 9 de Abril del mismo año, dos semanas que se corresponden con la época de obtención de polen en la zona. Cada dos días completos, se ha vaciado el contenido del cajoncillo del cazapolen, obteniéndose así unas muestras de cargas de polen, que seguidamente se ha desecado con aire caliente en una cámara secadora "Selecta", a $40^{\circ}$ C durante 2 horas ( Jean-Prost,1989). Las muestras, una vez desecadas, se han pesado en una balanza electrónica "Mettler PJ400" y envasado en botes herméticos que se han guardado en refrigerador $\left(4^{\circ} \mathrm{C}\right)$.

Dentro del período de muestreo, uno de los días (2-V) se han retirado las muestras cada dos horas, desde las 8 a.m. hasta las 8 p.m.

Aproximadamente en un radio de $2 \mathrm{Km}$., se han recolectado las plantas conocidas como melitófilas, además de las que se han observado que han sido visitadas por las abejas. Así mismo, se ha anotado la fenología floral de estas especies, siguiendo para ello la metodología de Anderson \& Hubricht, (1940). También se ha tomado nota del tipo de recompensa floral que obtiene la abeja y de la frecuencia subjetiva de estas especies en el territorio.

En cada muestra de cargas de polen, se ha efectuado una separación por el co-

lor y la textura de las cargas de polen. Previamente, hemos apartado una submuestra de 1 gr.del conjunto de cada muestra (Louveaux, 1958b). 
Para llevar a cabo el análisis cualitativo de los tipos polínicos presentes en cada muestra, primeramente se acetolizaron por separado una carga de cada submuestra "color-textura", siguiendo la metodología de Erdtman (1960) modificada por Reitsma (1969). En las preparaciones microscópicas así obtenidas, se procedió a la identificación de los tipos polínicos, para lo que se utilizaron las claves del Atlas Polínico de Andalucía Occidental (Valdés et al.1987), ayudándonos también de la palinoteca de referencia y de otros criterios como la fenología y distribución.

Con el fin de determinar los porcentajes de aparición de cada tipo polínico en cada una de las muestras, y al mismo tiempo detectar posibles especies enmascaradas por un mismo color de carga de polen, se procedió a la acetolisis de las submuestra de 1 gr, montándose cuatro preparaciones independientes de cada una. En cada preparación, se han contabilizado un mínimo de 300 granos de polen (Vergeron,1964). Para equilibrar las diferencias en volumen de los granos de polen de diferentes especies, se han calculado los porcentajes en volumen (\%V) (Louveaux,1958 b). Las clases de frecuencias consideradas han sido las de Zander (Sec. Maurizio \& Louveaux, 1967).

Algunas muestras se han preparado también para su observación al Microscopio Electrónico de Barrido, facilitándose en su caso la identificación de los táxones.

\section{R ES UL T A D O S Y D IS C U S IO N}

Se ha obtenido un total de 1310 gramos de polen de la colmena utilizada para el muestreo, cantidad que está comprendida en el promedio de polen cosechado en la zona.

Los resultados del análisis microscópico de las cargas de cada muestra se reflejan en la Tabla I. Se han detectado un total de 22 tipos polínicos correspondientes a 13 familias, pero la mayoría con escaso porcentaje de representación, siendo "raros" o "esporádicos".

Un 3\% de las cargas analizadas han resultado ser mixtas, compuestas por Convolvulus althaeoides y Lavandula stoechas en similar proporción, porcentaje algo mayor que los dados por otros autores (Shwan \& Martinons, 1954).

La mayor representatividad como pólenes "dominantes" en algunas muestras la tienen Cistus albidus y Citrus sp., con porcentajes que pueden llegar al 74\%.

En la Gráfica I se representan los tipos polínicos cuyo porcentaje de representación en el conjunto de polen recogido es mayor del 1\% (Lám. I). Se observa que Cistus albidus es la especie más recolectada por las abejas(43\%), seguida de Citrus sp. $(36 \%)$. En general, la familia Cistaceae supone un 55\% del recurso polínico en la zona. Esto significa que A. mellifera utiliza como fuente de polen sólo unas pocas especies, y que otras son utilizadas ocasionalmente. Siendo época primaveral, la selección de $A$. mellifera va a estar basada en la selección de aquellas especies que le sean más ventajosas (Louveaux, 1958a; Vissher \& Seseley, 1982) 
TIPOS POLINICOS

\section{CISTACEAE}

- Cistus albidus

- C. salvifolius

- C. ladanifer

- Fumana thymifolia

- T. Helianthemun salicifolium

(H. salicifolium, H. pillosum)

RUTACEAE

- Citrus sp. (C. auranthium,

(C. deliciosa, C. limetta,

C. sinensis, C. lemo)

FABACEAE

- Melilotus elegans

- T. Citysus scoparius

(U. baeticus, U. parviflorus,

Calicotome villosa)

- Vicia faba

ASTERACEAE

- Centaurea pullata

- T. Senecio vulgaris

(S. vulgaris, Carduus sp.,

Galactites tomentosa)

- Crepis vesicaria

VALERIANACEAE

- Fedia cornucopiae

LABIATEAE

- Lavandula stoechas

BORAGINACEAE

- Borago officinalis

- Echium plantagineum

OXALIDACEAE

- Oxalis pes-caprae

CONVOLVULACEAE

- Convolvulus althaeoides

GERANIACEAE

- Erodium cicutarium

MYRTACEAE

- Eucaliptus camadulensis

ROSACEAE

- Prunus domestica

LILIACEAE

- Asphodelus sp. \begin{tabular}{llllllll} 
M1 & M 2 & M 3 & M4 & M 5 & M 6 & M 7 & M 8 \\
\hline
\end{tabular}

$\begin{array}{cccccccc}66 & 69 & 34 & 63 & 44 & 11 & 20 & 16 \\ 11 & 16 & 11 & + & 3 & 3 & 8 & 5 \\ - & - & - & - & - & - & - & 3 \\ 2 & 2 & 12 & 11 & 2 & + & 6 & - \\ 1 & 1 & + & - & - & + & + & -\end{array}$

$\begin{array}{llllllll}15 & 4 & 13 & 15 & 39 & 74 & 56 & 73\end{array}$

$\begin{array}{llllllll}1 & 1 & 7 & 4 & 6 & 4 & 5 & 2 \\ 1 & 1 & 4 & 3 & 3 & + & 1 & 1\end{array}$

$\begin{array}{llllllll}2 & - & 6 & 2 & 1 & - & - & - \\ 1 & + & + & - & + & - & + & +\end{array}$

Tabla I. Espectro polínico del análisis cualitativo de las cargas de polen, expresado en \% V. El signo + se corresponde con los táxones con un porcentaje inferior al $1 \%$. Se subraya la especie más frecuente dentro de un tipo. M1= Muestra 1, etc. 
Cistus albidus

C. salvifolius

Fumana thymifolia

T. Helianthemum salicifolium

C. ladanifer

Prunus domestica

Citrus sp.

Yicia faba

Melilotus elegans

T. Citysus scoparius

Borago oficinalis

T. Senecio vulgaris

Senecio vulgaris

Carduus sp.

Galactites tomentosa

Centaurea pullata

Convolvulus althaeoides

Oxalis pes-caprae

Eucaliptus camadulensis

Lavandulas stoechas

Asphodelus ramosus

A. albus

Echium plantagineum

Erodium cicutarium

Fedia cornucopiae

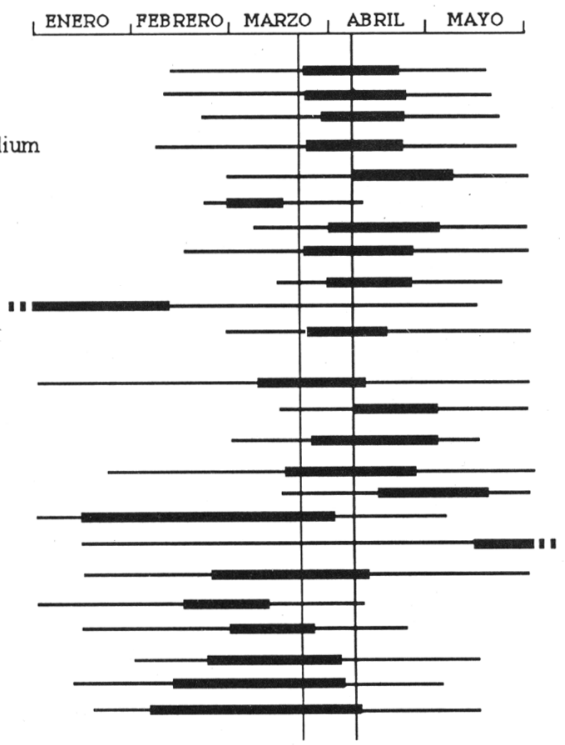

FRECUENCIA INTERES APICOLA

Tabla II. Datos sobre la fenología, frecuencia y recursos ofrecidos a A. mellifera de los tipos polínicos aparecidos en los análisis de las cargas polínicas (se subraya la especie más probable dentro de un tipo polínico). La trama gruesa se corresponde con el período de máxima floración; la trama más fina, con el inicio o final de la floración. 4= Muy Frecuente; $3=$ Frecuente; $2=$ Poco Frecuente; $1=$ Rara; += Muy Rara. N= Néctar; P=Polen. Las líneas verticales delimitan el periodo de estudio.

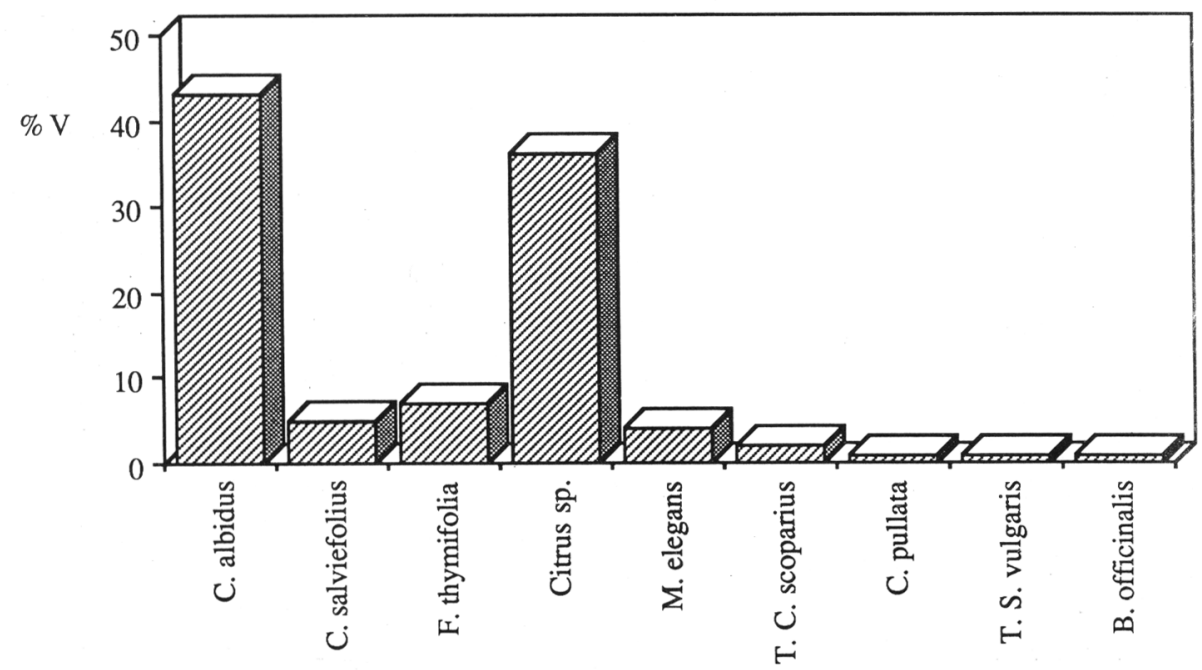

Gráfica I. Porcentajes de representación de cada tipo polínico en el total de polen recolectado durante el período de estudio. 
Cistus albidus, especie más utilizada en este caso, resulta ser una especie frecuente en la zona (Tabla II) y es de reconocer su tradición como buena fuente de polen (Sala, 1984; Bonet \& col., 1984, 1986; Talavera \& col., 1988). Al género Citrus pertenecen un conjunto de especies muy apetecidas también por A. mellifera en el territorio estudiado, debido quizá a su proximidad a la colmena y a que es muy frecuente (Tabla II). Además, una buena fuente de néctar, como es este caso, suele ser usada como aporte polínico (Percival, 1955):

Las leguminosas tales como M. elegans, U. baeticus y U. parviflorus, se comportan como significativas en la alternativa de recurso polínico. Estas especies son frecuentes en la zona, aunque en el caso de Ulex sp. (Tipo $C$.. scoparius), su importancia polinífera está disminuida debido a su estadío fenológico (Tabla II).

En la Tabla II vemos que, en general, la época de máxima floración de las especies correspondientes a los análisis de las cargas coincide con el período de muestreo. Estas especies suelen ser frecuentes en el territorio, y de un interés apícola basado en la oferta de polen (principalmente) y de néctar.

En la Gráfica II se presenta la variación en las proporciones de los tipos polínicos, mayormente representados, del período de muestreo. Cabe resaltar que C. albidus sigue una tónica general decreciente, pero manteniendose en unos porcentajes altos respecto a los demás tipos polínicos. Por el contrario, Citrus sp. sigue un patrón creciente durante el período de estudio, que coincide con el inicio de su máxima floración (Tabla II). Probablemente el comportamiento de esta especie sea la suplantación de $C$. albidus, ya que se encuentra más próxima a las colmenas y también es buena fuente néctar (Howes, 1953; Saury, 1981).

En la Gráfica III se representa la evolución individual durante una jornada de los tipos polínicos más representativos. El comportamiento general de todos es un aumento hacia el medio día y luego un descenso en las últimas horas. Sin embargo, M. elegans y Citrus sp. presentan una evolución contraria, aumentando su proporción en las últimas horas de la tarde, lo que probablemente se deba a que son especies muy próximas a las colmenas y están más asequibles para A. mellifera en las últimas salidas de pecoreo.

Las distintas horas de recogida de polen de las distintas especies se va a deber, principalmente, a la proximidad de la colmena, el tiempo de presentación y la competencia entre las especies (Percival, 1950, 1955). Entre las las 8 y las 10 de la mañana, se observa que hay una recogida mayoritaria de $C$. albidus y Citrus sp. y que luego a lo largo del día va aumentando la variabilidad en otras especies. Este comportamiento selectivo coincide con Percival (1947) el cual manifiesta que el polen recogido más temprano suele provenir de plantas próximas a la colmena y/o de las que están en lugares donde primero da el sol.

La variación del peso durante una jornada se representa en la Gráfica IV, estando la mayor recogida de polen en el intervalo de 10 a 12 horas. Como generalmente unas 2 horas después de la disponibilidad de polen en la planta se ejecuta la máxima actividad de recolección por las abejas (Percival, 1955), seguramente se corresponde con el máximo obtenido en dicho intervalo. 


\begin{tabular}{|c|c|c|}
\hline & PERIODO DE FLORACION & INTERES APICOLA \\
\hline \multicolumn{3}{|l|}{ LABIATEAE } \\
\hline - Rosmarinus officinalis & 31-III/1-X & $\mathbf{N}$ \\
\hline - Lavandula multifida & $13-$ VII/8-X & $\mathrm{N}$ \\
\hline - Teucrium fruticans & 7-II/14-IV & $\mathrm{N}$ \\
\hline - Salvia verbenaca & 10-II/15-III & $\mathrm{N}$ \\
\hline \multicolumn{3}{|l|}{ ASTERACEAE } \\
\hline - Calendulà arvensis & $7-\mathrm{X} / 22-\mathrm{IV}$ & $\mathrm{N} / \mathrm{P}$ \\
\hline - Calendula suffruticosa & $2-\mathrm{XII} / 30-\mathrm{V}$ & $\mathrm{N} / \mathrm{P}$ \\
\hline - Crepis vesicaria & 30-XII/7-V & $\mathrm{N} / \mathrm{P}$ \\
\hline - Reichardia picroides & 31-XI/7-IV & $\mathrm{N} / \mathrm{P}$ \\
\hline \multicolumn{3}{|l|}{ APOCINACEAE } \\
\hline - Vinca difformis & $1-X I / 23-V$ & $\mathrm{~N}$ \\
\hline \multicolumn{3}{|l|}{ ROSACEAE } \\
\hline - Prunus persica & 1-III/7-IV & $\mathrm{N} / \mathrm{P}$ \\
\hline - Cydonia oblonga & 7-III/9-IV & $\mathrm{N} / \mathrm{P}$ \\
\hline - Malus communis & $14-\mathrm{III} / 28-\mathrm{V}$ & $\mathrm{N} / \mathrm{P}$ \\
\hline \multicolumn{3}{|l|}{ CARYOPHYLACEAE } \\
\hline - Silene colorata & 14-I/7-IV & $\mathrm{N}$ \\
\hline - Arenaria hispanica & $21-\mathrm{I} / 23-\mathrm{IV}$ & $\mathrm{N}$ \\
\hline \multicolumn{3}{|l|}{ FABACEAE } \\
\hline - Adenocarpus telonensis & .14-III/1-VII & $\mathrm{N} / \mathrm{P}$ \\
\hline - Athyllis cytisoides & $1-I V / 20-V$ & $\mathrm{~N} / \mathrm{P}$ \\
\hline - Psoralea bituminosa & 14-III/23-VI & $\mathrm{N}$ \\
\hline - Retama sphaerocarpa & 21-IV/25-VI & $\mathrm{N}$ \\
\hline \multicolumn{3}{|l|}{ LHYTRACEAE } \\
\hline - Lhytrum junceum & 21-III/23-VI & $P$ \\
\hline \multicolumn{3}{|l|}{ BRASSICACEAE } \\
\hline - Brassica oleracea & 7-II/20-III & $\mathrm{N} / \mathrm{P}$ \\
\hline \multicolumn{3}{|l|}{ BORAGINACEAE } \\
\hline - Cynoglossum cheirifolium & 12-II/13-III & $\mathrm{N}$ \\
\hline - Anchusa azurea & 7-III/1-VIII & $\mathrm{N}$ \\
\hline \multicolumn{3}{|l|}{ LILIACEAE } \\
\hline - Allium triquetrum & 7-II/14-IV & $\mathrm{N} / \mathrm{P}$ \\
\hline - Ornithogalum narbonense & 7-III/1-V & $\mathrm{N}$ \\
\hline \multicolumn{3}{|l|}{ PAPAVERACEAE } \\
\hline - Papaver rhoeas & $13-\mathrm{II} / 30-\mathrm{V}$ & $P$ \\
\hline \multicolumn{3}{|l|}{ RANUNCULACEAE } \\
\hline - Anemone palmata & 7-II/14-IV & $\mathrm{P}$ \\
\hline \multicolumn{3}{|l|}{ RHAMNACEAE } \\
\hline - Rhamnus alaternus & 2-II/15-IV & $P$ \\
\hline \multicolumn{3}{|l|}{ CISTACEAE } \\
\hline - Cistus monspeliensis & $14-\mathrm{III} / 16-\mathrm{V}$ & $\mathrm{P}$ \\
\hline
\end{tabular}

Tabla III. Relación de otras especies de la zona que florecen en el período de estudio, y en las que la presencia de Apis mellifera ha sido observada. N= Néctar; $\mathrm{P}=$ Polen. 

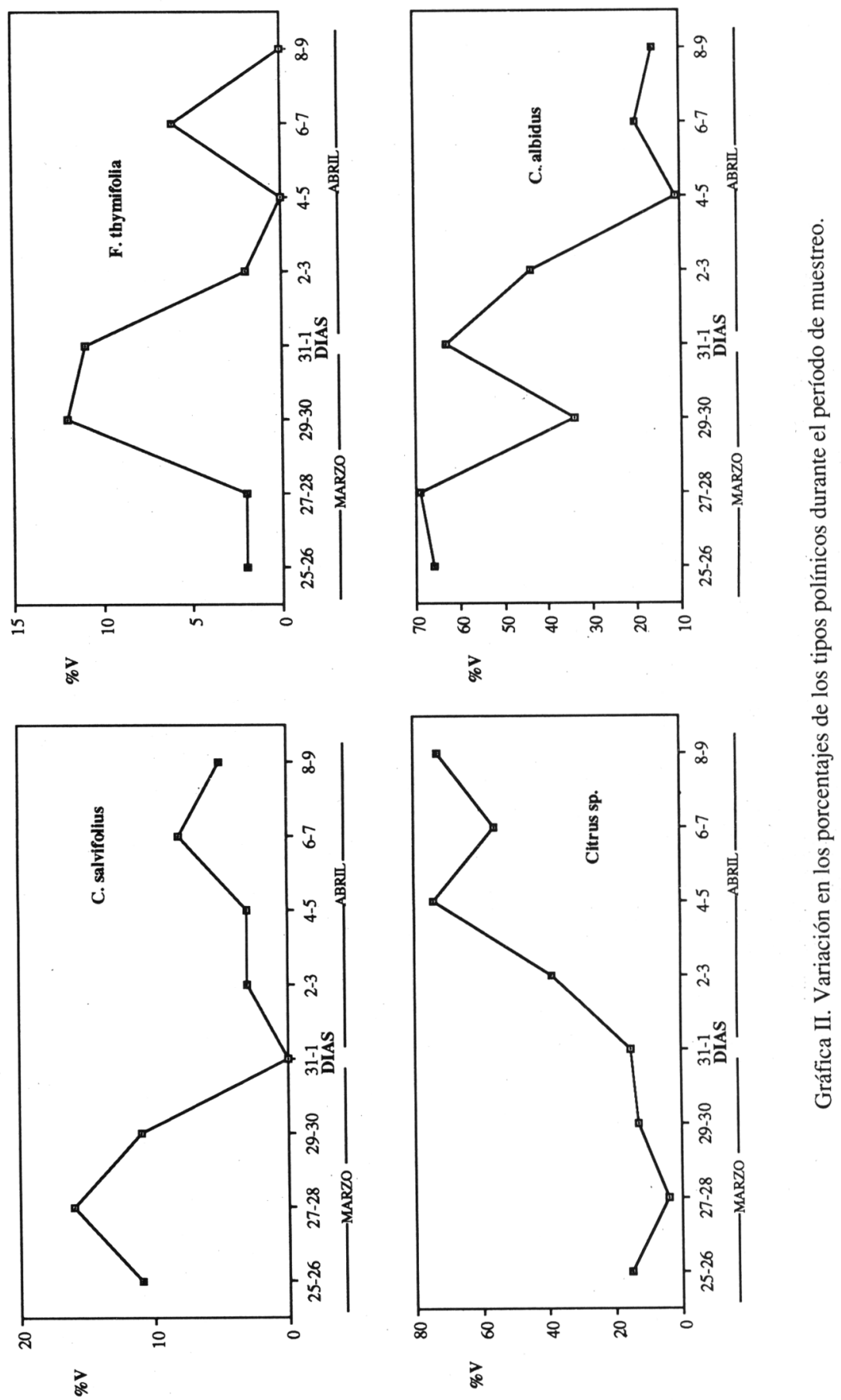

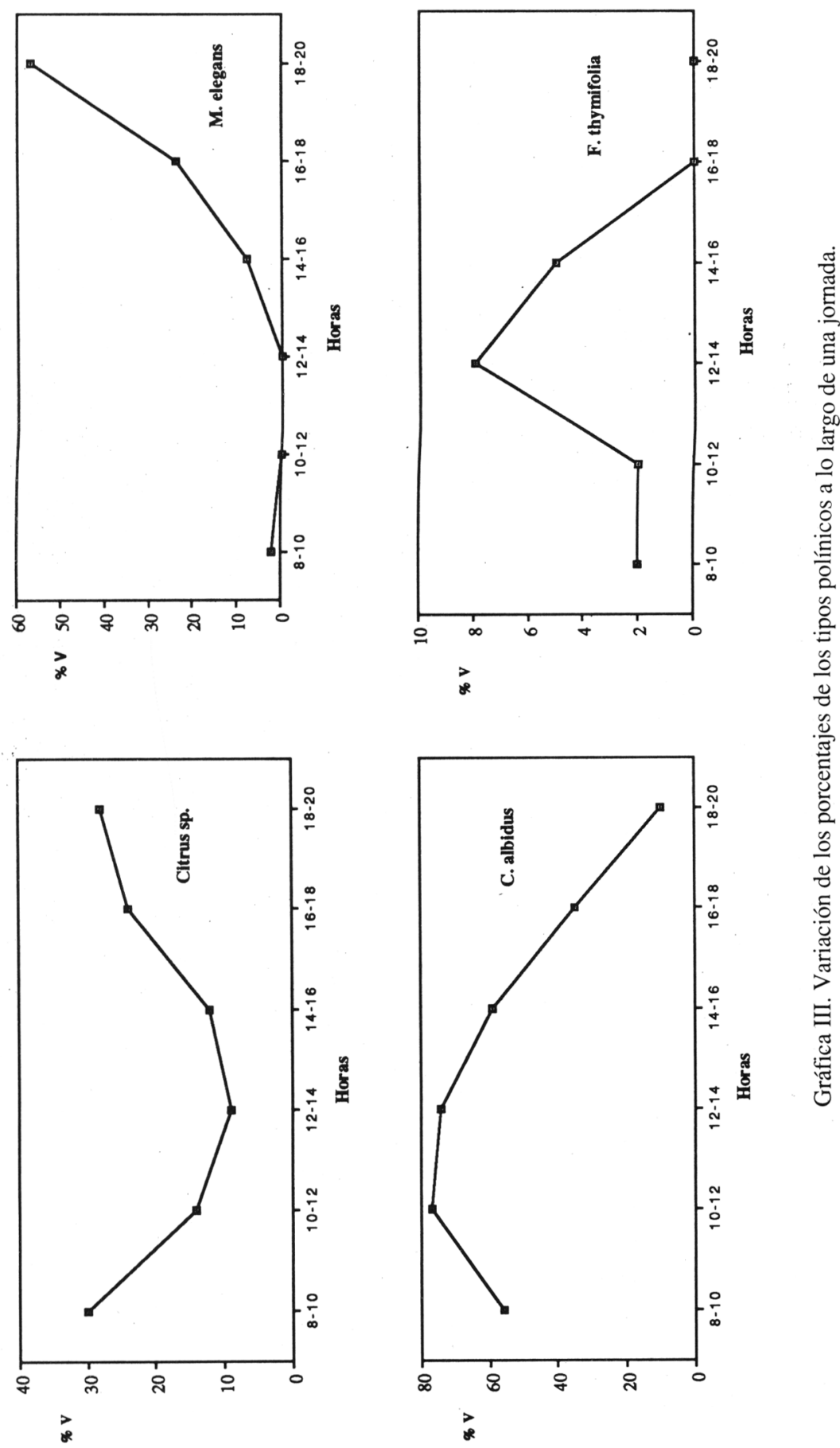


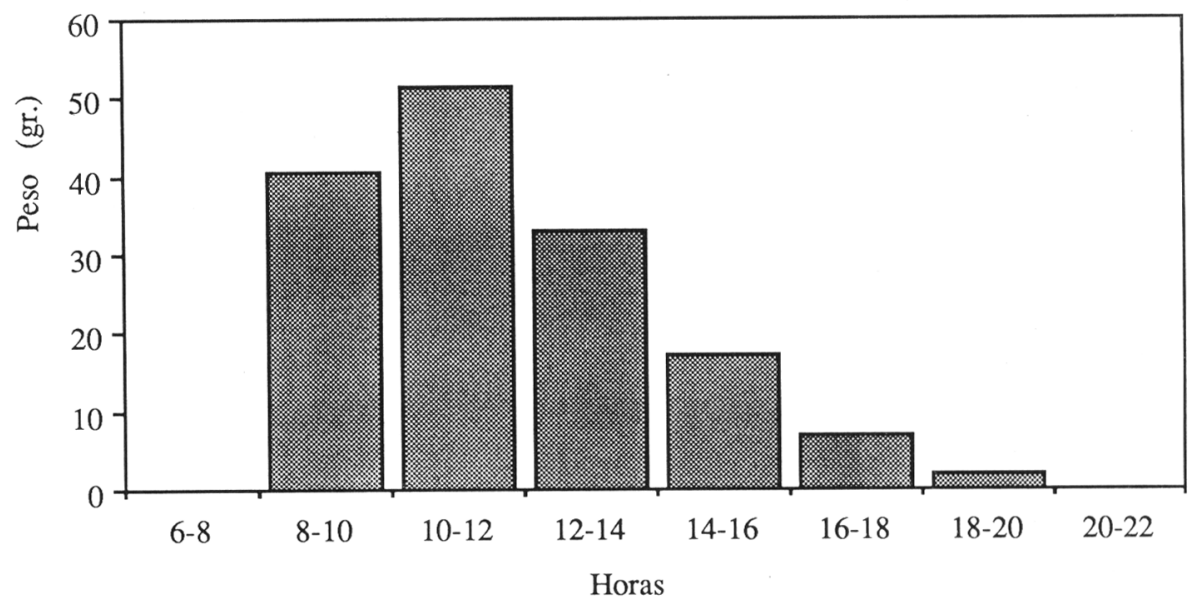

Gráfica IV. Variación del peso del polen recogido durante una jornada.

En la Tabla III, la relación de las especies que se presenta corresponde a las especies de la zona en las que se ha observado la presencia de A. mellifera recolectando néctar y/o polen y que no han aparecido en los análisis polínicos de las cargas, sin duda alguna porque se tratarían de especies visitadas por abejas de otras colmenas próximas.

AGRADECIMIENTOS. Los autores agradecen al Prof. Dr. B. Cabezudo y a la Dra. I. Fernández González sus sugerencias y ayuda.

\section{B I B L I O G R A F I A}

ANDERSON, E. \& HUBRICHT, L. -1940- A method or describing and comparing bloomingseasons. Bull. Torrey Bot. Club, 67(8):639-648.

BONET JORNET, A., SEBASTIA ALVAREZ, M.T. \& RITA LARRUCEA, J. -1984- La flora melífera de Barcelona. Vida apícola, 12:10-1.

BONET JORNET, A., SEBASTIA ALVAREZ, M.T. \& RITA LARRUCEA, J. -1986Principales floraciones melíferas de la provincia de Barcelona. Actas II Congr. Nac. Apic., (Gijón, 1984):109-117.

ERDTMAN, G. -1960- The acetolisis method. A revised description. Svensk. Bot. Tiaskr.,54:501-504.

GOMEZ FERRERAS, C. -1986- Origen botánico del polen comercializado en España. Actas II Congr Nac. Apic. (Gijón, 1984):70-93.

GONZALEZ BENAVENTE, F. -1984- El polen apícola español: composición botánica y características fisico-químicas. Actas I Congr. Nac. Apic. (Madrid, 1983): 31-44.

HOWES, F.N. -1953- Plantas melíferas. Ed. Reverté. Barcelona. 


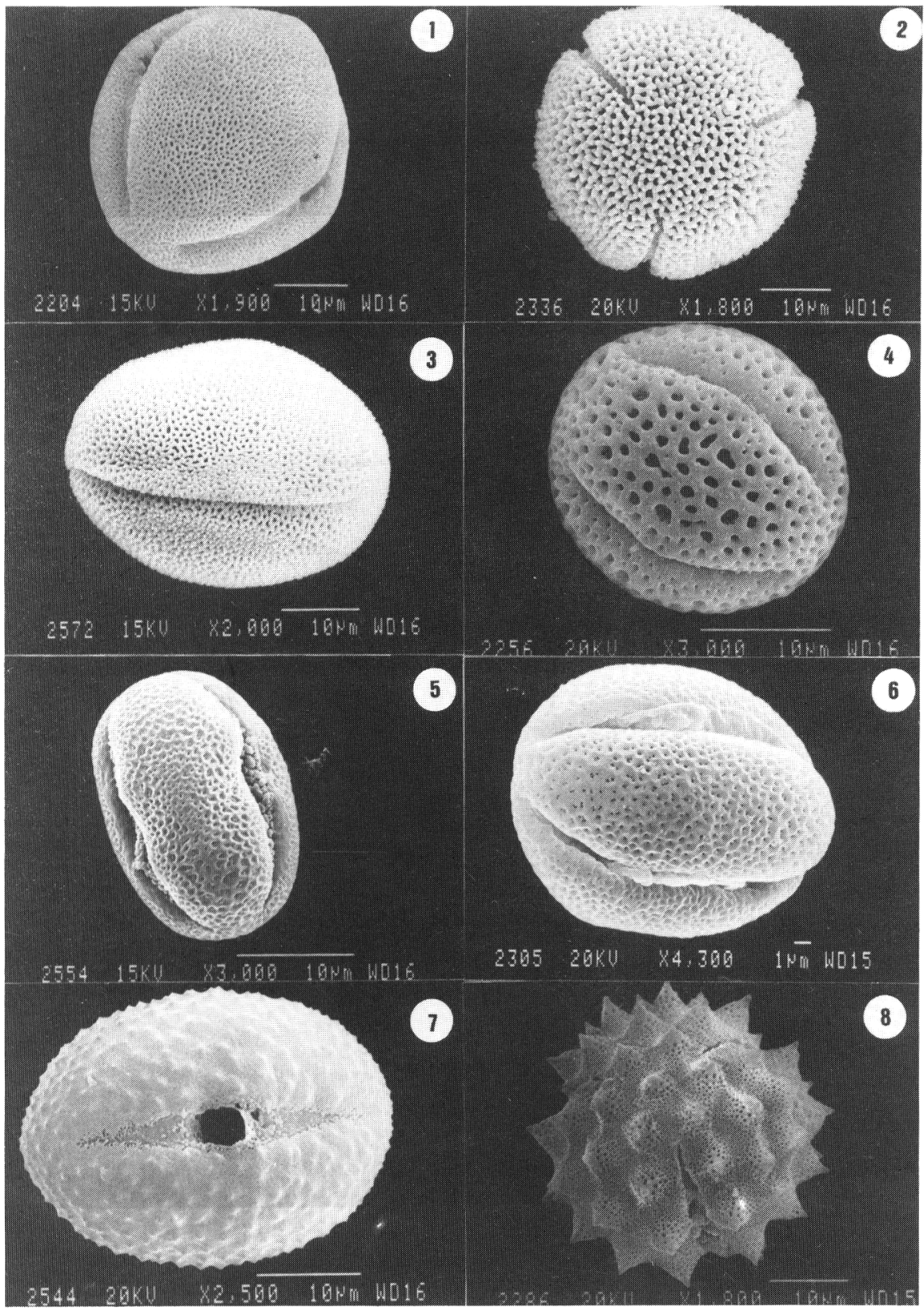

Lámina. I: Tipos polínicos más frecuentes que han aparecido en los análisis de las cargas de polen. 1: C. albidus; 2: C. salviefolius; 3: F. thymifolia; 4: Citrus sp.; 5: M. elegans; 6: T.C. scoparius; 7: C. pullata; 8: T.S. vulgaris. 
JEAN-PROST, P. -1989- Apicultura. Mundi Prensa. Madrid.

LOUVEAUX, J. -1958b- Recherches sur la récolte du pollen par les abeilles (Apis mellifera L.). Ann. Abeille 1 (III): 113-188.

LOUVEAUX, J. -1958c- Recherches sur la récolte du pollen par les abeilles (A. mellifera L.) (suite). Ann. Abeille I (IV): 197-221.

LOUVEAUX, J., MAURIZIO, A. \& VORWOHL, G. -1978- Methods of melissopalynology. Bee world, 59(4): 139-157.

MAURIZIO, A. \& LOUVEAUX, J. -1967- Les méthodes et la terminologie en melisopalynologie. Rev. Paleobot. Palynol., 3:291-295.

PERCIVAL, M. -1947- Pollen collection by Apis mellifera. New Phytol., 46-142-173.

PERCIVAL, M. -1950- Pollen presentation and collection New Phytol., 49:40-63.

PERCIVAL, M. - 1955- The presentation of pollen in certain Angiosperms and its collection by Apis mellifera. New Phytol., 54:353-368.

REITSMA, T.J. -1969- Size modification of recent pollen grains under different treatments. Rev. Paleobot. Palynol., 9:175-202.

SALA LLINARES, A. -1948- Plantas melíferas de la zona de Jijona (Alicante). Vida apícola, 11:52-56.

SAURY, A. -1981- Plantes melliferes. Ed. Lechevalier. Paris

SERRA, J. -1988- Origen botánico del polen apícola producido en España. Anales Asoc. Paolinol. Lengua Esp.: 4:73-78.

SERRA, J., GONELL GALINDO, \& GOMEZ PAJUELO, A. -1986c- El polen de abeja producido en España. Vida apícola, 19:35-38.

SHARMA, M. -1970- Analysis of pollen loads of honey bees from Kangra, India. Grana, 10:35-42.

SHWAN, B. \& MARTINO, S. -1954- Studier ovër binas (A. mellifera) pollen drag i Ultuna. Statens Hudsjursförsök Medelande,: 57.

SYNGE, A.D.-1947- Pollen collection by honey bees (Apis mellifera). Journal Animal Ecology, 16:122-138.

TAlaVERA, S., HERRERA, J., ARroyo, J., ORTIZ, P.L. \& DEVESA, J.A. -1988Estudio de la flora apícola de Andalucía Occidental. Lagascalia, 15 (extra): 567-591.

VALDES, B., DIEZ, M.J. \& FERNANDEZ, I. -1987- Atlas polínico de Andalucía Occidental. Inst. Des. Regional y Excma Diputación de Cádiz. Sevilla.

VERGERON, P. -1964- Interpretation statistique des résultats en matière d'analysis pollinique des miels. Ann. Abeille, 7(4):349-364.

VISSHER, P.K. \& SEELEY, T.D. -1982- Foraging strategy honey bee colonies in a temperature deciduous forest. Ecology, 63(6):1790-1801.

(Aceptado para su publicación el 20 de marzo de 1990)

Dirección de los autores: Departamento de Biología Vegetal. Facultad de Ciencias. Universidad de Málaga. 